\title{
The Potential Detrimental Role of High Salt Diet in Promoting Exaggerated Th-17 Responses in COVID-19 Patients, an Intriguing Hypothesis
}

\author{
Daniele Basta ${ }^{1,2, *}$ and Olga S Latinovic ${ }^{3,4}$ \\ ${ }^{1}$ Centro Universitario Sportivo-CUS, University of Calabria, Rende (CS), Italy \\ ${ }^{2}$ Associazione Biologi Nutrizionisti Calabresi-ABNC, Cosenza (CS), Italy \\ ${ }^{3}$ Institute of Human Virology, School of Medicine, University of Maryland, USA \\ ${ }^{4}$ Department of Microbiology and Immunology, School of Medicine, University of Maryland, USA
}

*Corresponding author: Daniele Basta, Ph.D, Associazione Biologi Nutrizionisti Calabresi, Centro Universitario Sportivo, University of Calabria, Via P. Bucci, Arcavacata di Rende (CS), 87036, Italy, Tel: +39 3404760323; E-mail: nutrizione.danielebasta@gmail.com

Received: 14 Apr, 2020 | Accepted: 14 Jul, 2020 | Published: 21 Jul, 2020

Citation: Basta D, Latinovic OS (2020) The Potential Detrimental Role of High Salt Diet in Promoting Exaggerated Th-17 Responses in COVID-19 Patients, an Intriguing Hypothesis. J Clin Lab Med 5(1): dx.doi.org/10.16966/2572-9578.135

Copyright: (C) 2020 Basta D, et al. This is an open-access article distributed under the terms of the Creative Commons Attribution License, which permits unrestricted use, distribution, and reproduction in any medium, provided the original author and source are credited.

\begin{abstract}
The novel coronavirus disease 2019 (COVID-19) is currently causing a global pandemic. The new Coronary Pneumonia originated in Wuhan and has spread rapidly throughout both China and most of the rest of the world (184 countries). The World Health Organization (WHO) declared COVID-19 a pandemic on March 11, 2020. In the absence of any pharmaceutical intervention, including prevention, treatment, and the cure, the only strategy against COVID-19 has been to reduce mixing susceptible and infectious people through early ascertainment of cases or to restrict physical contact. Social distancing is a necessary strategy as the best protection against further virus transmission and uncontrolled spread. Many COVID-19 patients develop Acute Respiratory Distress Syndrome (ARDS), which leads to pulmonary edema and lung failure. Patients may also undergo liver, heart, and kidney failure due to exuberant inflammatory responses during SARS-CoV-2 infection, further resulting in uncontrolled pulmonary inflammation, a likely leading cause of case fatality (Figure 1). The described clinical symptoms are associated with a "cytokine storm" involved in Th17-type exaggerated responses. The detrimental effects of a high-salt diet on human health have received much attention in the past few years. While it has been well established that high dietary salt intake is related to cardiovascular diseases, there is growing evidence that the excess of salt intake also induces IL-17-producing Th-17 cells originating from the gut compartment. It thus contributes to creating a pro-inflammatory microenvironment, predisposing or aggravating potential pulmonary ARDS complications in the increasing numbers of COVID-19 patients.
\end{abstract}

\section{Introduction}

The novel coronavirus disease (COVID-19, Coronavirus Disease-2019) is currently causing a pandemic affecting more than 13.5 million patients globally with 590,000 deaths [1]. The new Coronary Pneumonia emerged in China late in 2019 and rapidly spread around the world, becoming a global public health concern, mainly in the Middle East, Europe, and the USA, but affecting 184 countries worldwide. It is caused by SARS-CoV-2 (Severe Acute Respiratory Syndrome-Coronavirus-2) and has a case-fatality rate of 2-3\%, with higher rates among elderly patients and patients with comorbidities such as cardiovascular disease [2-6], diabetes [5-8], chronic respiratory disease $[5,6,8,9]$, hypertension $[5,6,10,11]$, and cancer [10-13]. The COVID-19 virus is severely challenging the Italian healthcare system and society, as the requests for intensive care treatment/prevention are much greater than the system's capacity under the overwhelming influx of infected patients. On March 11, 2020, the COVID-19 outbreak has been declared a pandemic by the
WHO. Since February 21, 2020, when the first case of COVID-19 was recorded in Italy, the National Healthcare Service, which offers universal access to health care, has faced increasing pressure, with more than 90,000 total cases of COVID-19 and more than 20,000 deaths as of April 10, 2020 [14]. In the absence of any pharmaceutical intervention, which includes prevention, treatment and cure, the only current strategy against COVID-19 is to reduce mixing of susceptible and infectious people through early ascertainment of cases and reduction of contact through social distancing. The social distancing was critical as it represented the best protection against further virus transmission and uncontrolled spread and it gave results in all affected areas in the world. It is important to recognize, however, that social distancing is not social isolation. For instance, doctors can practice telemedicine when following up with the patients. Regular office clerks, teachers, and students can practice teleworking and work or teach from home. We may also learn important lessons from previous failures in implementing a full national lock down in earlier times in some countries during previous pandemics. Part of the problem lies 


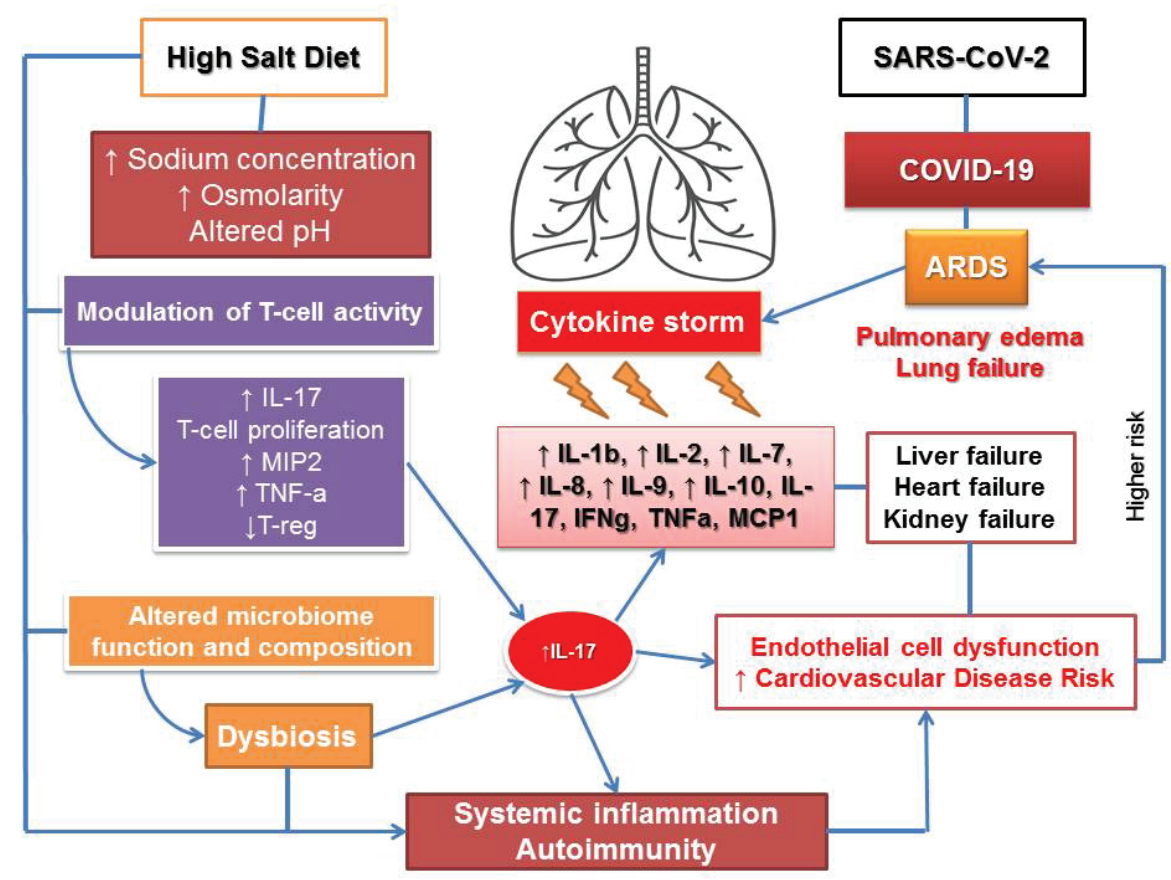

Figure 1: Potential effect of a high-salt diet on immune response in COVID-19.

in the lack of correct information and transparency about COVID-19 from China, where the virus originated presumably at the wet markets in Wuhan. Nevertheless, all data and information about the new Coronary Pneumonia in China can be obtained online in time. Another part lay in the lack of much knowledge about this novel virus, particularly its highly infectious nature and its serious pathology. This resulted in a serious lack of preparedness. Both factors lead to our current reality where COVID-19 has created great confusion and fear among the world's citizens. The consequences of the COVID-19 2020 pandemic will be tremendous, both medically and economically. The pandemic's impact on the world's economy is already estimated as higher than the Great Depression, with 17M lost jobs (mid April 2020 data) in the USA only [15].

Many COVID-19 patients develop Acute Respiratory Distress Syndrome (ARDS), which leads to pulmonary edema and lung failure. Patients may also undergo liver, heart, and kidney failure due to exuberant inflammatory responses during SARS-CoV-2 infection, further resulting in uncontrolled pulmonary inflammation, including Th-17-type responses, a likely leading cause of case fatality.

The negative effects of a high-salt diet on human health have received much attention in the past few years. While it has been well established that high dietary salt intake is related to cardiovascular diseases, there is growing evidence that the excess of salt intake also impairs immune system function and induces IL-17-producing Th-17 cells originating from the gut compartment. It thus could contribute to creating a pro-inflammatory microenvironment, predisposing or aggravating potential pulmonary ARDS complications in the increasing numbers of COVID-19 patients.

\section{Discussion}

Excess of dietary sodium that could cause an aggressive T-cell immune response in COVID-19 patients, aggravating or increasing the risk of cardiorespiratory complications. T-cell lymphocytes provide a variety of immune-related functions in controlling and shaping immune response. CD4+ T-cells and CD8+ T-cells in particular play a significant antiviral role by balancing the response to pathogens against the risk of developing autoimmunity or overwhelming inflammation. In conditions of chronic infection, persistent exposure of T-cells to high levels of antigen results in a severe T-cell dysfunctional state called exhaustion. T-cell exhaustion leads to suboptimal immunemediated control of multiple viral infections, including to the Human Immunodeficiency Virus (HIV-1) [16]. Chronic HIV-1 infection occurs in most patients in the presence of persistent high levels of viral replication [RNA load] and is associated with a loss of immune control over viral replication, partly due to depletion of the CD4+ T-cells. Also, however, with persistently high levels of viral antigen, HIV-1 specific T-cells become exhausted and lose their capacity to efficiently kill infected cells. In addition to high levels of viral antigen, the strong pro-inflammatory immune activation and compromised T-cell homeostasis during HIV-1 infection contribute to the development of T-cell exhaustion [17].

\section{Virus-induced ACE2 downregulation}

Many COVID-19 patients develop Acute Respiratory Distress Syndrome (ARDS), which leads to pulmonary edema and lung failure, and have liver, heart, and kidney damage $[5,18]$ due to exuberant inflammatory responses during SARS-CoV-2 infection. This damage further results in uncontrolled pulmonary inflammation, likely a leading cause of case fatality. Rapid viral replication and cellular damage, virus-induced ACE2 downregulation and shedding, and Antibody Dependent Enhancement (ADE) are responsible for aggressive inflammation caused by SARS-CoV-2 [19, 20]. The initial onset of rapid viral replication may cause massive epithelial and endothelial cell death and vascular leakage, triggering the overproduction of pro-inflammatory cytokines and chemokines. Loss of pulmonary ACE2 function has been proposed to be related to acute lung injury because ACE2 downregulation and shedding can lead 
to dysfunction of the Renin-Angiotensin System (RAS), and further enhance inflammation and cause vascular permeability [21]. From a pathological point, severe COVID-19 patients showed pulmonary bilateral diffuse alveolar damage with cellular fibromyxoid exudates [22]. Interstitial mononuclear inflammatory infiltrates, dominated by lymphocytes, could be observed in both lungs [22]. Multinucleated syncytial cells with atypical enlarged pneumocytes characterized by large nuclei, amphiphilic granular cytoplasm, and prominent nucleoli were identified in the intra-alveolar spaces, indicating viral cytopathiclike changes [22]. Massive mucus secretion in both lungs was found in victims of COVID-19, different from the situation in SARS and MERS $[20,22]$.

\section{Cytokine storm}

Theearlierdescribed clinicalsymptomsare associated witha "cytokine storm" [23], manifesting in elevated serum levels of Interleukin-1 beta (IL-1b), Interleukin-2 (IL-2), Interleukin-7 (IL-7), Interleukin-8 (IL8), Interleukin-9 (IL-9), Interleukin-10 (IL-10), Interleukin-17 (IL17), Granulocyte Colony-Stimulating Factor (G-CSF), GranulocyteMacrophage Colony-Stimulating Factor [GM-CSF], Interferon gamma (IFN- $\gamma$ ), Tumor Necrosis Factor Alpha (TNF- $\alpha$ ), Interferon $\gamma$-induced Protein (IP10), Monocyte Chemoattractant Protein-1 (MCP1), Macrophage Inflammatory Protein-1-alpha (MIP1A), and Macrophage Inflammatory Protein-1-beta (MIP1B) [23]. Compared with non-ICU patients, ICU patients have even higher levels of IL-2, IL-7, IL-10, G-CSF, IP10, MCP1, MIP1A, and TNF- $\alpha$ [24]. Amongst these, several cytokines are involved in Th-17-type responses. IL-1b and TNF- $\alpha$ (Th-17 and Th1 cells both highly express TNF- $\alpha$ ), promote Th-17 responses and vascular permeability and leakage [24]. IL-17 has broad pro-inflammatory effects on induction of cytokines G-CSF (responsible for granulopoiesis and recruitment of neutrophils). IL$1 b$, IL-6, and TNF- $\alpha$ can cause systemic inflammatory symptoms, including fever. IL-17 and GM-CSF are associated with autoimmune and inflammatory diseases [25].

$\mathrm{Xu} \mathrm{Z}$, et al. [18] showed that the peripheral blood of a patient with severe COVID-19 had a strikingly high number of CCR6+Th17 cells, further supporting the involvement of a Th-17-type cytokine storm in this disease. Elevated Th-17 (as well as Th1) responses or enhanced IL17-related pathways are also observed in MERS-CoV and SARS-CoV patients $[26,27]$. Taken together, the Th-17-type response contributes to the "cytokine storm" in pulmonary viral infection including SARSCoV-2, which results in tissue damage and likely promotes pulmonary edema. Targeting the Th17 pathway may benefit the patients with Th17-dominant immune profiles. Current evidence strongly indicates that the Th1-type response is key for successful control of SARS-CoV and MERS-CoV [26-30], and this is probably true for SARS-CoV-2 as well. The CD8+ T-cell response, even though crucial, needs to be well controlled in order not to cause lung pathology. Repurposing currently marketed drugs would provide valuable opportunities, because it will take a couple of years to develop new specific drugs to treat COVID-19.

This "cytokine storm" can initiate viral sepsis and inflammatoryinduced lung injury, which then leads to other complications including Pneumonitis, ARDS, respiratory failure, septic shock, lungs failure, and potentially death in high risk groups, as discussed earlier [30,31]. Further autopsy or biopsy studies are necessary to better understand details of this disease and its severity (virulent nature, infectious rate 10x higher than flu). These findings are in line with SARS and MERS in that the presence of lymphopenia and the "cytokine storm" may have a major role in the pathogenesis of COVID-19 [31,26], but further investigations in clinical research, basic science, and epidemiology are badly needed.

\section{Th-17 cells and cytokine storm}

CD4+ T-cells (including Th1, Th2, Th-17, and Treg) contribute to both immunopathology and viral clearance of influenza infection $[32,33]$. Severe respiratory influenza is often characterized by the early secretion of Th1 and Th-17 cytokines [34]. Accumulating data suggest that Th-17 cells play a significant role in infectious diseases, autoimmune conditions, adoptive immune responses, and mucosal immunity [34,35]. For example, Th-17 immunity participates in clearing pathogens during host defense, but is also involved in tissue inflammation (for example, in several autoimmune diseases, allergic diseases, and asthma) [36-38]. An increase in IFN- $\gamma$ IL-8, IL-9, IL13 and IL-10 in both critical and non-critical hospitalized H1N1infected patients compared to those with mild symptoms indicates that elevated cytokine levels constitute hallmarks of severe H1N1 disease [31]. Another argument supporting the hypothesis that the Th-17 inflammatory response is the origin of the severe respiratory disease caused by H1N1 infection is that high levels of Th-1- and Th17- based mediators accompany the disease [31]. Alternatively, an increase in Th-1 and Th-17 cytokines may reflect a vigorous antiviral host response necessary for clearance of virus during severe lower respiratory infections [31]. While the ability of influenza A virus to induce the production of chemotactic (RANTES, MIP-1 $\alpha$, MCP-1, MCP3, and IP-10) and pro-inflammatory (IL-1 $\beta$, IL-6, IL-18, and TNF- $\alpha$ ) Th-1-related mediators is well known from previous reports on seasonal influenza, Bermejo-Martin JF, et al. [31] reported the first evidence that the Th-17 response is a signature of severe influenza disease in humans. In addition to preclinical results, recent clinical data show a correlation between enhanced IL-17 production and increased frequencies of Th-17 cells in many human autoimmune diseases, including Multiple Sclerosis, Rheumatoid arthritis, Systemic Lupus Erythematosus, Psoriasis, Inflammatory Bowel Disease, and Crohn's disease [39].

Th-17 cells themselves produce IL-17, GM-CSF, IL-21 and IL22. IL-17 has broad pro-inflammatory effects through induction of production of cytokines G-CSF (responsible for granulopoiesis and recruitment of neutrophils), IL-1 $\beta$, IL-6, TNFa; chemokines KC, MIP2A, IL-8, IP10, MIP3A (which attract and recruit more immune infiltrates); and matrix metalloproteinases (participating in tissue damage and remodeling) [36,37]. IL-17 and GM-CSF plays essential roles in driving autoimmune inflammation in mice [40]. IL-21 is required for Th-17 cell maintenance and germinal center responses in a STAT3 dependent manner. Xu Z, et al. [18] showed that peripheral blood of a patient with severe COVID-19 had a strikingly high number of CCR4+CCR6+ Th-17 cells, further supporting a TH17 type cytokine storm in this disease. Moreover, there is evidence that Th-17 cytokines may contribute to airway fibrosis during asthma by enhancing production of eosinophil derived pro-fibrotic cytokines [41].

\section{Potential therapeutic treatments against SARS-CoV-2}

Due to the rapid diffusion of SARS-CoV-2 in 2020, it's crucial to identify effective treatment options. In this regard, drug remdesivir has received particular attention in the last two months. Remdesivir represents a nucleotide analog drug that exhibit antiviral effects against SARS-CoV-2 through many potential mechanisms, including nsp12 polymerase activity interfering and viral replication inhibition. Moreover, remdesivir can maintain its therapeutic effectiveness even due to the existent high genetic barrier as a difficult obstacle for coronavirus to achieve drug resistance. According to Grein J, et al., [42] $68 \%$ of the patients receiving, at least one dose of remdesivir, showed certain clinical improvements, including oxygen-status and 
extubation from mechanical ventilation. That resulted in a lower mortality rate.

Besides antiviral drugs, hydroxychloroquine (HCQ) and chloroquine (CQ) are known for their use against malaria and certain inflammatory conditions, such as rheumatoid arthritis, systemic lupus erythematosus, and porphyria cutanea tarda. Both drugs have in vitro activity against SARS-CoV, SARS-CoV-2, and other coronaviruses, with hydroxychloroquine having relatively higher potency against SARS-CoV-2 $[43,44]$. One small study reported that hydroxychloroquine alone or in combination with azithromycin reduced detection of SARS-CoV-2 RNA in upper respiratory tract specimens compared with a non-randomized control group but did not assess clinical benefit [45]. Nevertheless more thorough clinical studies are required to fully elucidate a possibility of CQ and HCQ therapeutic effectiveness. Both CQ and HCQ are weak bases that are known to elevate the $\mathrm{pH}$ of acidic intracellular organelles, such as endosomes and lysosomes essential for membrane fusion. Since acidification is crucial for endosome maturation and function, the investigators surmised that endosome maturation might be blocked at intermediate stages of endocytosis, resulting in a failure of further viral transport to the ultimate releasing site [46].

\section{Salt and immune response}

The effect of alterations in extracellular $\mathrm{pH}$ on cellular and humoral immune function has been studied since the 1950s. The maintenance of a resting intracellular $\mathrm{pH}$ of 6.8-7.3 and alterations in intracellular $\mathrm{pH}$ accompanying the cellular events outlined are considered primarily to be a result of an energy-requiring, active proton extrusion system. A major mechanism for active $\mathrm{H}+$ extrusion from the plasma membrane of a large variety of cells is the carrier-mediated $\mathrm{Na}+\mathrm{H}+$ exchanger NHE-1 (anti-port, counter-transport system) that catalyzes the exchange of sodium for hydrogen. This $\mathrm{Na}+\mathrm{-H}+$ exchanger constitutes a form of secondary, active transport, relying on the steep, extracellular $\mathrm{Na}+$ gradient provided by the primary $\mathrm{Na}+\mathrm{K}+$ ATPase pump to extrude one hydrogen ion from the cytosol in exchange for the entry of one $\mathrm{Na}+$. It is also capable of mediating the exchange of a narrow selection of other monovalent ions, for example external $\mathrm{H}+$ for internal $\mathrm{Na}+$, external $\mathrm{Li}$ for internal $\mathrm{H}+$, external $\mathrm{H}+$ for internal $\mathrm{Li}$, and $\mathrm{Na}+$ for $\mathrm{Na}+$. The primary physiological role of this exchanger is to maintain intracellular $\mathrm{pH}$ homeostasis by extruding metabolically generated $\mathrm{H}+$ ions $[47,48]$. Innate and adaptive immune cells can sense hypertonic sodium in the interstitial tissues, which subsequently affects their differentiation and/or function [49].

It is well know that sodium is an indispensable nutrient for proper cell functions when consumed in appropriate amounts [50]. Sodium is essential for maintenance of osmotic pressure, normal $\mathrm{pH}$, distribution of body fluids, and for most metabolic processes [49]. Sodium chloride is readily available in processed food and ingested in large amounts as part of Western diets. The salt content in processed foods can be more than 100 times higher in comparison to similar home-made meals [51,52].

High salt intake represents a risk factor for hypertension, a condition related to higher mortality rate in COVID-19 patients. Salt sensitivity is a complex phenomenon for which blood pressure changes can occur on the basis of dietary salt intake and it can vary from person to person. Genetic and environmental factors can contribute to salt-sensitivity. According to the GenSalt study about $39 \%$ of Chinese adults, one of the most affected populations by the new coronary pneumonia, are salt-sensitive [53].
The detrimental effects of a high-salt diet on human health have received much attention in the past few years. While it has been well established that high dietary salt intake is related to cardiovascular diseases, there is growing evidence that excess salt also affects the immune system [54,55].

Elevated concentration of sodium chloride was found to induce proinflammatory IL-17-producing Th17 cells via the p38/MAPK/nuclear factor of activated T-cells pathway and the serum/glucocorticoidregulated kinase 1 /forkhead box protein 1 [SGK1] pathway both in vitro and in vivo [56].

The molecular mechanisms underlying the sensing of sodium ions and the initial activation of the Th-17 cells have been largely elucidated. Hypertonic salt directly activates the kinase SGK1, which stabilizes interleukin-23R and thus reinforces the Th-17 phenotype [56]. It also activates other molecules that induce the expression of SGK1. It seems that high-salt conditions are needed in the lymphoid tissues for an optimal adaptive immune response during infection. In contrast, and as shown in table 1 . The relatively low sodium concentration in blood may provide protection against systemic Th-17-driven inflammation [55].

T-cell function is also affected by sodium concentration [57,58]. Increasing $\mathrm{NaCl}$ concentration by $\approx 40 \mathrm{mmol} / \mathrm{L}$ boosts IL- 2 expression and consequently T-cell proliferation. Furthermore, hypertonic saline added to the culture media may help restore the function of inhibited T-cells [59]. In addition to stimulating T-cell proliferation, salt suppresses anti-inflammatory activity [60], promotes the production of MIP-2 (macrophage inflammatory protein-2) and TNF- $\alpha$, and synergistically increases MIP- 2 production induced by lipopolysaccharide or TNF- $\alpha$ [61].

It has recently been reported that a high sodium diet leads to profound immune changes in the gut compartment. Increased exposure to dietary salt causes an increased inflammatory milieu within the gastrointestinal tract [55]. Dietary changes may also influence both the function and composition of the gut microbiome, which in turn could impact both innate and adaptive immunity through inducing increases of inflammatory cells while causing loss of T-reg function $[62,63]$. In the gut, induction of Th-17 cells by high salt intake depends on altered compositions of the gut microbiota. Junger WG, et al. [58] demonstrated that a moderate high-salt challenge reduced intestinal survival of Lactobacillus spp., increased Th-17 cells, and increased blood pressure. This result confirms and highlights the gut microbiome as a potential therapeutic target to counteract saltsensitive conditions.

A salt rich diet induces the accumulation of T-helper lymphocytes in the gut when producing the pro-inflammatory cytokine interleukin-17 (Th-17) [61-63]. These changes in the gut have been shown to promote autoimmunity and exacerbate experimental allergic encephalomyelitis, an animal model of multiple sclerosis $[64,65]$.

Table 1: Effects of sodium chloride on different immune cells (60).

\begin{tabular}{|l|c|l|}
\hline \multicolumn{1}{|c|}{ Cell type } & $\begin{array}{c}\text { Effector function } \\
\text { under high-salt }\end{array}$ & \multicolumn{1}{|c|}{ Molecular mechanism } \\
\hline M1-type macrophage & $\uparrow$ & p38-MAPK, NFAT-5 \\
\hline M2-type macrophage & $\downarrow$ & mTOR, Akt \\
\hline T-reg & $\downarrow$ & Sgk-1 \\
\hline Th-17 & $\uparrow$ & p38-MAPK NFAT-5, Sgk-1 \\
\hline Th-2 & $\downarrow$ & N.D. \\
\hline Th-1 & $\uparrow$ & N.D. \\
\hline DC & $\uparrow$ & p38-MAPK, Sgk-1 \\
\hline
\end{tabular}


Neutrophil infiltration in the colonic mucosa is one of the distinguishing characteristics of acute inflammation present in inflammatory bowel disease and correlates with disease severity [66]. Neutrophil lifespan is tightly regulated in order to limit unintended damage to tissues by secreted reactive oxygen species and granular enzymes meant to degrade extracellular matrices and disrupt tight junctions. Increased neutrophil lifespan is observed under inflammatory conditions and has been attributed to both direct interaction with microbes and the release of cytokines from other immune cells $[67,68]$. In particular, Hensley-McBain T, et al. [68] recently reported that a reduced Lactobacillus: Prevotella ratio is associated with neutrophil survival, suggesting that intestinal bacteria may contribute to Gastrointestinal Compartment (GI) neutrophil accumulation in treated HIV-1 infection.

In a recent study, Faraco G, et al. [69] revealed a new gut-brain axis linking high-salt diet to cognitive impairment through a gutinitiated adaptive immune response compromising brain function via circulating IL-17. They reported that, in mice, excess dietary salt suppresses resting cerebral blood flow and endothelial function, leading to cognitive impairment. This effect depends on expansion of Th-17 cells in the small intestine, resulting in a marked increase in plasma IL-17. Circulating IL-17, in turn, further promotes endothelial dysfunction and cognitive impairment by the Rho kinase-dependent inhibitory phosphorylation of endothelial nitric oxide synthase and reduced nitric oxide production in cerebral endothelial cells.

Considering that IL-17 is potentially vasotoxic, these observations raise the solid hypothesis that the Th-17 response induced by high dietary salt could play a significant role in the attendant vascular dysfunction and respiratory complications in COVID-19 patients. For example, Yi B, et al. [70] used an experimental approach of fixing the salt intake of healthy human subjects at amounts of 12,9 , and $6 \mathrm{~g} / \mathrm{d}$ for 2 months and examined the relationship between salt intake levels and changes in the immune system. It is well known that excess dietary sodium predisposes to high blood pressure and is associated with an increased cardiovascular risk, conditions related to severe COVID-19 complications. Their results showed that subjects on the high-salt diet of $12 \mathrm{~g} / \mathrm{d}$ displayed a significantly higher number of immune cell monocytes compared with the same subjects on a lower-salt diet, and a correlation test revealed a strong positive association between salt intake levels and monocyte numbers, confirming once again that the decrease in salt intake was accompanied by reduced production of pro-inflammatory cytokines IL-6 and IL-23, along with enhanced production of the anti-inflammatory cytokine IL-10.

\section{Conclusions}

Altogether these data suggest that a high-salt diet in COVID-19 patients may have detrimental effects, inducing differentiation and exaggerated Th-17 responses, and aggravating the related "cytokine storm", potentially starting from the gut microenvironment. Although more data and clinical trials are needed to confirm this hypothesis, we believe it is important to minimize salt intake during the quarantine by reducing highly processed food, canned food, processed nitratecontaining meat products, and other food high in sodium. This could be important in avoiding potentially aggressive Th-17 pro-inflammatory responses, aggravating potential pulmonary ARDS complications in the currently increasing numbers of COVID-19 patients. This pandemic is a huge burden on health care workers worldwide and its resolution is critical for the good of our communities, our countries, and our world.

\section{Conflict of Interest Statement}

The authors declare that they have no conflict of interest.

\section{Acknowledgement}

The authors would like to thank Prof. Marvin Reitz, PhD, Dr Yutaka Tagaya, PhD, MD, and Matthew Weichseldorfer, MSc from the Institute of Human Virology, University of Maryland School of Medicine in Baltimore, MD for their critical review and valuable comments on the manuscript.

\section{References}

1. WHO (2020) WHO statement on cases of COVID-19 surpassing 100000 .

2. Clerkin JK, Fried JA, Raikhelkar J, Sayer G, Griffin JM, et al. (2020) Coronavirus Disease 2019 (COVID-19) and Cardiovascular Disease. Circulation 141: 20

3. Bonow RO, Fonarow GC, O'Gara PT, Yancy CW, et al. (2020) Association of Coronavirus Disease 2019 (COVID-19) With Myocardial Injury and Mortality. JAMA Cardiol.

4. Guo T, Fan Y, Chen M, Wu X, Zhang L, et al. (2020) Cardiovascular Implications of Fatal Outcomes of Patients With Coronavirus Disease 2019 (COVID-19). JAMA Cardiol : e201017.

5. Huang C, Wang Y, Li X, Ren L, Zhao J, et al. (2020) Clinical features of patients infected with 2019 novel coronavirus in Wuhan, China. The Lancet 395: 497-506.

6. Wu Z, McGoogan JM (2020) Characteristics of and Important Lessons From the Coronavirus Disease 2019 (COVID-19) Outbreak in China: Summary of a Report of 72314 Cases From the Chinese Center for Disease Control and Prevention. JAMA.

7. Fadini GP, Morieri ML, Longato E, Avogaro A (2020) Prevalence and impact of diabetes among people infected with SARS-CoV-2. J Endocrinol Invest 28: 1-3.

8. Zhou F, Yu T, Du R, Fan G, Liu Y, et al. (2020) Clinical course and risk factors for mortality of adult inpatients with COVID-19 in Wuhan, China: a retrospective cohort study. Lancet 395: 1054-1062.

9. Lippi G, Henry BM (2020) Chronic obstructive pulmonary disease is associated with severe coronavirus disease 2019 (COVID-19). Respir Med 167: 105941.

10. Lippi G, Wong J, Henry BM (2020) Hypertension and its severity or mortality in Coronavirus Disease 2019 (COVID-19): a pooled analysis. Pol Arch Intern Med.

11. Fang L, Karakiulakis G, Roth M (2020) Are patients with hypertension and diabetes mellitus at increased risk for COVID-19 infection? Lancet Respir Med 8: e21.

12. Zhang L, Zhu F, Xie L, Wang C, Chen R, et al. (2020) Clinical characteristics of COVID-19-infected cancer patients: A retrospective case study in three hospitals within Wuhan, China. Ann Oncol 31: 894-901.

13. Liang W, Guan W, Chen R, Wang W, Li J, et al. (2020) Cancer patients in SARS-CoV-2 infection: a nationwide analysis in China. Lancet Oncol 21: 335-337.

14. Ministry of Health of Italy (2020) COVID-19-situazione in Italia.

15. WolfersJ (2020) The Unemployment Rate is Probably Around 13 Percent. The New York Times.

16. Fenwick $C$, Joo V, Jacquier $P$, Noto $A$, Banga $R$, et al. (2019) T-cell exhaustion in HIV infection. Immunol Rev 292: 149-163. 
17. Papagno L, Spina CA, Marchant A, Salio M, Rufer N, et al. (2004) Immune activation and CD8+ T-cell differentiation towards senescence in HIV-1 infection. PLoS Biol 2: e20.

18. Xu Z, Shi L, Wang Y, Zhang J, Huang L, et al. (2020) Pathological findings of COVID-19 associated with acute respiratory distress syndrome. Lancet Respir Med 8: 420-422.

19. Imai Y, Kuba K, Penninger JM, (2008) The discovery of angiotensinconverting enzyme 2 and its role in acute lung injury in mice. Exp Physiol 93: 543-548.

20. Jin Y, Yang H, Ji W, Wu W, Chen A, et al. (2020) Virology, Epidemiology, Pathogenesis, and Control of COVID-19. Viruses 12: 372.

21. Yang M (2020) Cell Pyroptosis, a Potential Pathogenic Mechanism of 2019-nCoV Infection. SSRN

22. Liu Q, et al. (2020) Anatomy of a COVID-19 Death Corpse System. J Forensic Med 36: 21-23.

23. Canna SW, Behrens EM (2012) Making sense of the cytokine storm: a conceptual framework for understanding, diagnosing, and treating hemophagocytic syndromes. Pediatr Clin North Am 59: 329-344.

24. Tse GM, To KF, Chan PKS, Lo AWI, Wu A, et al. (2004) Pulmonary pathological features in coronavirus associated severe acute respiratory syndrome (SARS). J Clin Pathol 57: 260-265.

25. Wong CK, Lam CWK, Wu AKL, Ip WK, Lee NLS, et al. (2004) Plasma inflammatory cytokines and chemokines in severe acute respiratory syndrome. Clin Exp Immunol 136: 95-103.

26. Josset L, Menachery VD, Gralinski LE, Agnihothram S, Sova P, et al. (2013) Cell Host Response to Infection with Novel Human Coronavirus EMC Predicts Potential Antivirals and Important Differences with SARS Coronavirus. mBio 4: e00165-00113.

27. Faure E, Poissy J, Goffard A, Fournier C, Kipnis E, et al., Distinct immune response in two MERS-CoV-infected patients: can we go from bench to bedside? PLoS One 9: e88716.

28. Wu D, Yang XO (2020) TH17 responses in cytokine storm of COVID-19: An emerging target of JAK2 inhibitor Fedratinib. J Microbiol Immunol Infect 53: 368-370.

29. Bermejo-Martin, Jesus F, Pumarola T, Rello J, Almansa R, et al. (2009) Th1 and Th17 hypercytokinemia as early host response signature in severe pandemic influenza. Crit Care13: R201.

30. Mahallawi WH, Khabour OF, Zhang Q, Makdoum HM, Suliman BA, et al. (2018) MERS-CoV infection in humans is associated with a proinflammatory Th1 and Th17 cytokine profile. Cytokine 104: 8-13.

31. Nicholls JM, Poon LLM, Lee KC, Ng WF, Lai ST, et al. (2003) Lung pathology of fatal severe acute respiratory syndrome. Lancet 361 : 1773-1778.

32. Bhattacharyya Mitra, Madden P, Henning N, Gregory S, Aid M, Martinot AJ, et al. (2017) Regulation of CD4 T cells and their effects on immunopathological inflammation following viral infection. Immunol 152: 328-343.

33. McKinstry KK, Strutt TM, Swain SL (2011) Hallmarks of CD4 T cell immunity against influenza. J Intern Med 269: 507-518.

34. Ouyang W, Kolls JK, Zheng Y (2008) The biological functions of T helper 17 cell effector cytokines in inflammation. Immunity 28: 454 467.

35. Tesmer LA, Lundy SK, Sarkar S, Fox DA (2008) Th17 cells in human disease. Immunol Rev 223: 87-113.
36. Hoe E, Anderson J, Nathanielsz J, Toh ZQ, Marimla R, et al. (2017) The contrasting roles of Th17 immunity in human health and disease. Microbiol Immunol 6: 49-56.

37. Korn T, Bettelli E, Oukka M, Kuchroo VK (2009) IL-17 and Th17 cell. Annu Rev Immunol 27: 485-517.

38. Louten J, Boniface K, de Waal Malefyt R (2009) Development and function of TH17 cells in health and disease. J Allergy Clin Immunol 123: $1004-1011$.

39. Waite JC, Skokos D (2012) $\mathrm{Th}_{17}$ response and inflammatory autoimmune diseases. Int J Inflam 2012: 819467.

40. Kuwabara T, Ishikawa F, Kondo M, Kakiuchi T (2017) The Role of IL17 and Related Cytokines in Inflammatory Autoimmune Diseases. Mediatiators Inflamm 2017: 3908061.

41. Al-Muhsen S, Letuve S, Vazquez-Tello A, Pureza MA, Al-Jahdali H, et al. (2013) Th17 cytokines induce pro-fibrotic cytokines release from human eosinophils. Respi res 14: 34.

42. Grein J, Ohmagari N, Shin D, Diaz G, Asperges E, et al. (2020) Compassionate use of remdesivir for patients with severe Covid-19. N Engl J Med 382: 2327-2336.

43. Colson P, Rolain JM, Lagier JC, Brouqui P, Raoult D (2020) Chloroquine and hydroxychloroquine as available weapons to fight COVID-19. Int J Antimicrob Agents 4:105932.

44. Yao X, Ye F, Zhang M, Cui C, Huang B, et al. (2020) In Vitro Antiviral Activity and Projection of Optimized Dosing Design of Hydroxychloroquine for the Treatment of Severe Acute Respiratory Syndrome Coronavirus 2 (SARS-CoV-2). Clin Infect Dis 2020: ciaa237.

45. Gautret P, Lagier JC, Parola P, Hoang VT, Meddeb L, et al. (2020) Hydroxychloroquine and azithromycin as a treatment of COVID-19: results of an open-label non-randomized clinical trial. Int J Antimicrob Agents 2020: 105949.

46. Mauthe M. Orhon I, Rocchi C, Zhou X, Luhr M, et al. (2018). Chloroquine inhibits autophagic flux by decreasing autophagosomelysosome fusion. Autophagy 14: 1435-1455.

47. Mahnensmith TL, Aronson PS (1985) The plasma membrane sodium-hydrogen exchanger and its role in physiological and pathophysiological processes. Circ Res 56: 773-788.

48. Lardner A (2001) The effects of extracellular $\mathrm{pH}$ on immune function. J Leukoc Biol 69: 522-530.

49. Wilck N, Balogh A, Markó L, Bartolomaeus H, Müller DN (2019) The role of sodium in modulating immune cell function. Nat Rev Nephro 15: $546-558$

50. Farquhar WB, Edwards DG, Jurkovitz CT, Weintraub WS (2015) Dietary sodium and health: more than just blood pressure. J Am Coll Cardiol 65:1042-1050.

51. Appel LJ, Frohlich ED, Hall JE, Pearson TA, Sacco RL, et al. (2011) The importance of population-wide sodium reduction as a means to prevent cardiovascular disease and stroke: a call to action from the American Heart Association. Circulation 123: 1138-1143.

52. Brown IJ, Tzoulaki I, Candeias V, Elliott P (2009) Salt intakes around the world: implications for public health. J Epidemiol 38: 791-813.

53. GenSalt Collaborative Research Group (2007) GenSalt: rationale, design, methods and baseline characteristics of study participants. J Hum Hypertens 21: 639-646.

54. Kong YW, Baqar S, Jerums G, Ekinci El (2016) Sodium and Its Role in Cardiovascular Disease-The Debate Continues. Front Endocrinol (Lausanne) 7: 164. 
55. Willebrand R, Kleinewietfeld M (2018) The role of salt for immune cell function and disease Immunology 154: 346-353.

56. Wu C, Yosef N, Thalhamer T, Zhu C, Xiao S, et al. (2013) Induction of pathogenic TH17 cells by inducible salt-sensing kinase SGK1. Nature 496: 513-517.

57. van der Meer JW, Netea MG (2013) A Salty Taste to Autoimmunity. J New England J Med 2520-2521.

58. Junger WG, Hoyt DB, Hamreus M, Liu FC, Herdon-Remelius C, et al. (1997) Hypertonic saline activates protein tyrosine kinases and mitogen-activated protein kinase p38 in T-cells. J Trauma 42: 437 443.

59. Wilck N, Matus MG, Kearney SM, Olesen SW, Forslund K, et al. (2017) Salt-responsive gut commensal modulates $\mathrm{TH}_{17}$ axis and disease. Nature 551: 585-589.

60. Hernandez AL, Kitz A, Wu C, Lowther DE, Rodriguez DM, et al. (2015) Sodium chloride inhibits the suppressive function of FOXP ${ }^{3+}$ regulatory T cells. J Clin Invest 125: 4212-4222.

61. Kostyk AG, Dahl KM, Wynes MW, Whittaker LA, Weiss DJ, et al. (2006) Regulation of chemokine expression by $\mathrm{NaCl}$ occurs independently of cystic fibrosis transmembrane conductance regulator in macrophages. Am J Pathol 169: 12-20.

62. Kleinewietfeld M. Manzel A, Titze J, Kvakan H, Yosef N, et al. (2013) Sodium chloride drives autoimmune disease by the induction of pathogenic $\mathrm{TH}_{17}$ cells. Nature 496: 518-522.
63. Vieira SM, Pagovich OE, Kriegel MA (2014) Diet, microbiota and autoimmune diseases. Lupus 23: 518-526.

64. Honda K, Littman DR (2012) The microbiome in infectious disease and inflammation. Annu Rev Immunol 30: 759-795.

65. Muthas D, Reznichenko A, Balendran CA, Böttcher G, Clausen IG et al. (2017) Neutrophils in ulcerative colitis: a review of selected biomarkers and their potential therapeutic implications. Scand J Gastroenterol 52: 125-135.

66. Sabroe I, Prince LR, Jones EC, Horsburgh MJ, Foster SJ, et al. (2003) Selective roles for Toll-like receptor (TLR) 2 and $T_{4} R_{4}$ in the regulation of neutrophil activation and life span. J Immunol 170: 5268-5275.

67. Colotta F, Re F, Polentarutti N, Sozzani S, Mantovani A (1992) Modulation of granulocyte survival and programmed cell death by cytokines and bacterial products. Blood 80: 2012-2020.

68. Hensley-McBain T, Wu MC, Manuzak JA, Cheu RK, Gustin A, et al. (2019) Increased mucosal neutrophil survival is associated with altered microbiota in HIV infection. PLoS Pathog 15: e1007672.

69. Faraco G, Brea D, Garcia-Bonilla L, Wang G, Racchumi G, et al. (2018) Dietary salt promotes neurovascular and cognitive dysfunction through a gut-initiated $\mathrm{TH}_{17}$ response. Nat Neurosci 21: 240-249.

70. Yi B, Titze J, Rykova M, Feuerecker M, Vassilieva G, et al. (2015) Effects of dietary salt levels on monocytic cells and immune responses in healthy human subjects: a longitudinal study. Transl Res 166: 103-110. 